\title{
COVID-19: How to select patients for endoscopy and how to reschedule the procedures?
}

\section{다)(우우}

\author{
Authors \\ Thierry Ponchon ${ }^{1}$, Stanislas Chaussade ${ }^{2}$ \\ Institutions \\ 1 Edouard Herriot Hospital - Hepatogastroenterology, \\ Lyon, France \\ 2 Cochin Hospital - Gastroenterology, Paris, France \\ Bibliography \\ DOI https://doi.org/10.1055/a-1154-8768 | \\ Endoscopy International Open 2020; 08: E699-E700 \\ (c) Georg Thieme Verlag KG Stuttgart · New York \\ eISSN 2196-9736
}

\author{
Corresponding author \\ Thierry Ponchon, Edouard Herriot Hospital - \\ Hepatogastroenterology, Place Arsonval, Lyon 69003, \\ France \\ Fax: + 33472110147 \\ thierry.ponchon@chu-lyon.fr
}

The COVID-19 epidemic that continues to progress requires an almost daily reflection on the different recommendations that can be made. Several recommendations or statements have already been posted or published in order to ensure safety against the virus in endoscopy units before, during, and after procedures.

Two other important questions related to the COVID-19 epidemic should also be considered: How to reduce the workload in endoscopy? and How to reschedule the cases that have been postponed?

The survey reported by Bilal et al. deals with the first subject. Of note is that the method used for the survey is original and adapted to this period of epidemic: The survey was conducted on Twitter using the "Twitter poll" option. As acknowledged by the authors, the sample is certainly not representative $(2,500$ followers of the author) but the idea is interesting.

First issue: Why and how to reduce the workload in endoscopy? In other words, why and how should we reconsider the indications?

Why reconsider the indications? The main reasons for this delicate question are:

- To reduce risk of healthcare personnel and patient exposure to the infection of the virus, which is present in saliva, expectorations and feces.

- To reduce risk to patients associated with transportation to the hospital and stay at the hospital, because hospitals also receive patients suspected of being infected with COVID-19.

- To avoid a shortage of personal protective equipment (PPE) for personnel who are in charge of taking care of patients with COVID-19. Unfortunately, this is probably the main reason in many countries, including so-called developed countries. This is a major drawback related to globalization.
For example, two-thirds of medical gloves are produced in only one country (Malaysia). It is also due to a lack of anticipation of the epidemic by the health authorities.

- To avoid having to manage complications of endoscopy that may require surgery and resuscitation at a time when intensive care unit beds have to be prioritized for patients with COVID-19.

- To reallocate human resources (nurses, anesthesiologists) and sedatives in order to increase the number of intensive care beds.

How to reconsider the indications?

This is the hardest part, and the paper by Bilal et al illustrates the variability in the opinion of endoscopists on this subject.

The usual indications for emergency endoscopy, digestive or biliopancreatic, are of course to be maintained and we will not discuss them. Palliative procedures intended to bypass digestive or biliary obstacles in highly symptomatic patients can be included in this category.

The question is whether to postpone non-urgent endoscopies when doing so would result in a lost chance for the patient, such as:

- all diagnostic techniques for neoplastic lesions (suspected neoplastic lesion, tissue acquisition, loco-regional staging)

- all therapeutic techniques for resection of neoplastic lesions including superficial cancers.

Two indications, in particular, are emblematic of this gray zone and of the difficulty in making recommendations: iron deficiency anemia and, above all, positive fecal immunohistochemistry testing (FIT). These patients agreed to testing and will have to wait for colonoscopy. In fact, the issues vary from one territory 
to another, and are evolving according to the scale of the crisis and, therefore, over time. What is certain? The decision should be made at the regional level and not at the level of a single institution, as the potential and residual means should be shared at this level. Then the complex equation should take into account the quantities of PPE available (the more important key factor); the state of saturation of intensive care units; potential length of time the procedure would be postponed (we can envision at least 2 months of delay); and the potential risk of loss of the chance in the specific case. We suggest that each institution - or better, each region - define a precise algorithm and for some cases, organize a consensus meeting with other colleagues about which endoscopic procedures to postpone and which to perform.

Furthermore, patients should be clearly informed about the reasons for postponing an endoscopy and the decisions should be documented in their files and in a letter to the referring doctors

It would be useful for national societies to produce standard documents to guide the response of endoscopy operators and to underscore, at a national level, any medicolegal issues.

Second issue: How to reschedule cases that have been postponed?

When normal activities resume, avoiding an overload of endoscopy units will be the primary concern. In fact, during the pandemic, 2 to 3 months of activity will have been lost while the incidence of digestive pathologies and the total number of patients will not have changed.

It is probably not necessary to give patients new appointments immediately because the duration of the confinement period for COVID-19 is uncertain. The risk may be in postponing a patient's endoscopy appointment twice. We suggest establishing several lists of patients whose appointments need to be rescheduled.

We also suggest not having a single list with all patients on it. In our units, we created several lists, each of which corresponded to a different level of priority for rescheduling, taking into account indications for endoscopy. The number of lists and their contents for an endoscopy unit, therefore, would depend on that unit's recruitment typology. The priority list must include patients with the highest risk of loss of chance, depending upon their history, macroscopic pattern of the lesion, and histology. That list might include, for example, patients with colorectal non granular lateral spreading adenomas; patients with superficial esophageal or gastric carcinoma; and those who have a positive FIT test and pancreatic nodules. Within each list, patients could be arranged in chronological order to reduce delays in scheduling overall.

In summary, the current priority that is quite appropriately being given to patients with COVID-19 should not result in a loss of continuity of care for other patients who need endoscopies. A minimum of collective (regional, if possible) organizational and common rules for endoscopy are necessary so that our patients do not suffer extensively from postponements of examinations, especially given that the duration of confinement remains uncertain.

\section{Competing interests}

The authors declare that they have no conflict of interest. 